\title{
Pandemic influenza $A(H 1 N 1) 2009$ virus outbreak among boarding school pupils in Madagascar: compliance and adverse effects of prophylactic oseltamivir treatment
}

\author{
Soatiana Rajatonirina ${ }^{1}$, Jean-Michel Heraud ${ }^{2}$, Laurence Randrianasolo ${ }^{1}$, Norosoa \\ Razanajatovo ${ }^{2}$, Tombo Ramandimbisoa ${ }^{3}$, Maherisoa Ratsitorahina ${ }^{1}$ and Vincent Richard ${ }^{1}$ \\ ${ }^{1}$ Epidemiologic unit, Institut Pasteur from Madagascar, Antananarivo, Madagascar \\ ${ }^{2}$ National Influenza Center, Virology Unit, Institut Pasteur from Madagascar, Antananarivo, Madagascar \\ ${ }^{3}$ School health service, Antananarivo, Madagascar
}

\begin{abstract}
Introduction: In October 2009, the first outbreak of pandemic influenza A(H1N1) 2009 virus in Madagascar occurred at a school in Antananarivo. Among the first 12 cases, five were reported in boarding pupils at the school. The school closed 10 days into the outbreak. Mass oseltamivir prophylactic treatment was used to contain the outbreak. This study aimed to determine the transmission of infection among boarding school pupils and to evaluate the adverse effects of oseltamivir chemoprophylactic treatment and their impact on compliance.

Methodology: After conducting an initial investigation of the outbreak we administered a questionnaire to 132 boarders who were present after the school re-opened. Questions addressed symptoms of influenza-like illness, compliance with chemoprophylaxis, and adverse effects. Results: Of 59 boarders, $20(45.0 \%)$ had confirmed pandemic influenza A (H1N1) infection. Among the asymptomatic boarders, compliance with oseltamivir chemoprophylaxis was moderate: $56.2 \%$ took the full 10 -day course, and $66.9 \%$ completed at least seven days. In contrast, among symptomatic boarders, only two did not take the full course of oseltamivir. Fifty percent of the boarders receiving oseltamivir experienced symptoms such as fatigue (38.7\%), difficulty concentrating (22.6\%) and headaches (19.4\%). Bad compliance was not associated with adverse effects.

Conclusion: Since the symptoms of pandemic influenza A(H1N1) 2009 virus were generally mild, the burden of adverse effects must be considered when deciding on mass oseltamivir chemoprophylaxis among teenagers.
\end{abstract}

Key words: Influenza A(H1N1)v; boarders; Madagascar

J Infect Dev Ctries 2011; 5(3):156-162.

(Received 23 June 2010 - Accepted 02 August 2010)

Copyright ( 92011 Rajatonirina et al. This is an open-access article distributed under the Creative Commons Attribution License, which permits unrestricted use, distribution, and reproduction in any medium, provided the original work is properly cited.

\section{Introduction}

Annually, there are one million deaths from influenza worldwide [1], and this incidence is higher during pandemics [2]. Since the first human cases of pandemic influenza A(H1N1) 2009 virus were identified in Mexico and the United States, the infection spread rapidly worldwide. The first cases of pandemic influenza $\mathrm{A}(\mathrm{H} 1 \mathrm{~N} 1) 2009$ infection in Madagascar were confirmed in August 2009 and by October 2009, only 16 cases were reported, all occurring in patients who had travelled to affected areas (internal communication from the Malagasy National Influenza Center).

Since April 2009, different countries have had many outbreaks within schools [3-6]. In some countries $[4,6]$, closing affected schools and offering antiviral prophylaxis with oseltamivir were the initial policies. Other countries decided to close schools when there was a marked increase in hospitalization or when school operations were affected by absenteeism [5]. Social distancing interventions such as school closure are among the initial means to control the epidemic spread of a novel influenza virus $[7,8]$. The risk of disease transmission may be further reduced by antiviral prophylactic treatments such as oseltamivir. However, limited evidence is available concerning the effectiveness of these measures during a real outbreak $[9,10]$.

The objectives of this study were to determine the extent of pandemic influenza A(H1N1) 2009 virus transmission among boarding school pupils, and to evaluate the adverse effects of oseltamivir chemoprophylactic treatment and their impact on compliance to help establish appropriate management strategies for boarding schools. 


\section{Methodology}

An outbreak of pandemic 2009 influenza infection occurred at a school in Antananarivo, Madagascar, with a population of 1,941 consisting of 314 staff members and 1,627 pupils including 143 boarders. The first cases of pandemic influenza A(H1N1) 2009 infection were identified on Friday, 8 October, one week before the school holidays. The outbreak subsequently spread among boarders during the weekend. Because school closure was not decided, the following control measures were implemented particularly for boarders: survey for active case finding, treatment for infected pupils, and prophylaxis for contacts. Subsequent cases were confirmed in all classes of the school. After informing parents by email and cellular phone (SMS), infected day pupils were evicted from the school. A recommendation previously had been given to parents to keep day pupils at home if ill.

Parents of boarders were informed about antiviral prophylaxis and were asked for written consent. Boarders were offered prophylaxis with oseltamivir $75 \mathrm{mg}$ once daily for 10 days starting on 12 October. Confirmed cases of pandemic influenza $A(\mathrm{H} 1 \mathrm{~N} 1)$ 2009 infection among boarders were isolated and offered treatment with oseltamivir $150 \mathrm{mg}$ once daily for five days.

An assessment and collection point was established at the school to assess and offer treatment to all pupils, staff members, and families. The school was closed for holidays from 16 October until 1 November. A second survey was administered to evaluate the compliance of antiviral prophylaxis or treatment, and the adverse effects among boarders who had been given prophylaxis.

\section{Case definition}

During the outbreak period, swabs were taken mostly from boarders who had respiratory symptoms such as cough, shortness of breath, sore throat and nasal discharge with and without fever.

Pandemic influenza A(H1N1) 2009 infection was confirmed by real-time RT-PCR using a panel of oligonucleotide primers and dual labeled hydrolysis (Taqman $\left.{ }^{\circledR}\right)$ probes (InfA, swInfA, swH1) according with The CDC Realtime RTPCR (rRTPCR) Protocol for Detection and Characterization of Swine Influenza[11].

\section{Compliance and adverse effects study}

An anonymous questionnaire designed by the Institut Pasteur from Madagascar was given to boarders to obtain demographic, epidemiologic, and clinical information on illness and adverse effects after taking oseltamivir. This was completed in writing on 2 November under supervision. Parents were informed about the questionnaire and were free to opt out of its completion.

\section{Statistical analysis}

Data were entered using double data entry into a relational ACCESS database and checked for errors. Statistical analyses were performed with $\mathrm{R}$ software [12]. Descriptive analyses were comprised of frequency distributions and proportions for each variable category. Group comparisons were performed with Fisher's exact test for categorical variables and ANOVA for continuous variables. $\mathrm{P}$ values were two-sided.

Logistic regression analysis was performed to measure the association between compliance and each independent variable. Odds ratios (OR) and $95 \%$ confidence intervals $(\mathrm{CI})$ were calculated from $\beta$ coefficients and their standard errors.

\section{Ethical clearance}

The study was approved by the Ministry of Health and the National Ethics Committee of Madagascar. Informed consent was obtained from at least one parent of each child.

\section{Results}

\section{Description of the outbreak among boarders}

The pupil presenting on 6 October complained only of cough without fever or other symptoms. A history of fever plus two or more other relevant influenza-like illness (ILI) symptoms did not start in the boarders' population before the first confirmed case on 8 October.

Nasopharyngeal swabs were collected from three boarders who visited the school sickroom on 8 October complaining of ILI symptoms. Laboratory analyses confirmed pandemic influenza $\mathrm{A}(\mathrm{H} 1 \mathrm{~N} 1)$ 2009 infection. Other pupils in the same school but from different classes also tested positive for pandemic influenza $A(H 1 N 1) 2009$ infection on the same day.

Between 6 October and 2 November, there were 59 cases with at least one influenza-like symptom among the 132 boarders (Figure1). Nasopharyngeal swabs were taken from 42 symptomatic cases $(71.2 \%)$ during the outbreak while of the rest of the 17 cases, six were no longer symptomatic at the time the school was opened, one was symptomatic before 
Figure 1. Numbers of symptomatic cases on a day-by-day basis

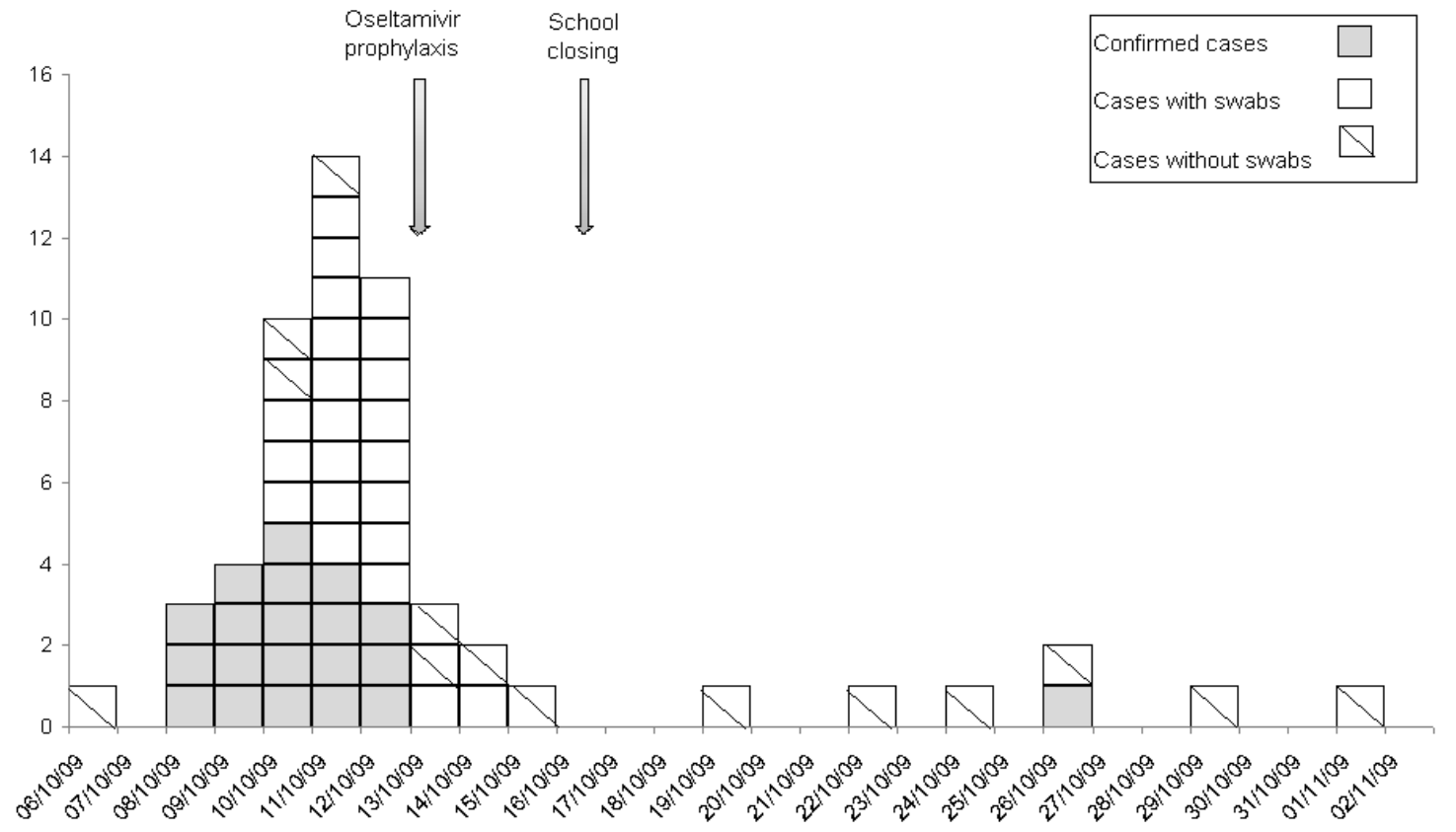

the outbreak was recognized, seven did not visit the school infirmary, and three had developed the illness at an unknown date. Of 42 symptomatic cases, pandemic influenza $\mathrm{A}(\mathrm{H} 1 \mathrm{~N} 1) 2009$ infection was confirmed in 20 cases $(47.6 \%)$.

\section{Clinical epidemiology}

Information on the prevalence of ILI symptoms among boarders was obtained the week before school closure and during the holidays. Boarders with clinical or confirmed pandemic influenza $A(\mathrm{H} 1 \mathrm{~N} 1)$ 2009 infection were notified during sampling and other pupils were notified during retrospective interviews. The distribution of symptoms among confirmed and clinical cases is shown in Table 1. Confirmed cases showed a distribution of ILI symptoms such as cough (85.0\%), sore throat $(70.0 \%)$, and nasal discharge $(70.0 \%)$. There were no hospitalized cases. Information on the duration of symptoms was not available.

\section{Compliance with prophylaxis}

The questionnaire was given to all 132 (92\%) boarders present at school on 2 November (71 girls and 61 boys mean age 16.9 years). All boarders completed the questionnaire. All confirmed cases were offered antiviral treatment and other boarders were offered antiviral prophylaxis.

Among the 20 boarders who were pandemic influenza $\mathrm{A}(\mathrm{H} 1 \mathrm{~N} 1) 2009$ positive, only two (10.0\%) did not take the full course of oseltamivir treatment (Figure 2) while four took a prolonged course ( $>5$

Table 1. Clinical manifestations of symptomatic patients

\begin{tabular}{|c|c|c|c|c|c|c|c|}
\hline & \multicolumn{2}{|c|}{$\begin{array}{c}\text { H1N1v Positive } \\
\text { (1) }\end{array}$} & \multicolumn{2}{|c|}{$\begin{array}{c}\text { H1N1v Negative } \\
\text { (2) }\end{array}$} & \multicolumn{2}{|c|}{$\begin{array}{c}\text { Cases with ILI (Without lab } \\
\text { confirmation) } \\
(3)\end{array}$} & \multirow[t]{2}{*}{ P-value } \\
\hline & $\mathrm{n}=20$ & $(\%)$ & $n=22$ & $(\%)$ & $\mathrm{n}=17$ & $(\%)$ & \\
\hline Cough & 17 & $(85.0)$ & 8 & $(36.4)$ & 11 & $(64,7)$ & 0.82 \\
\hline Sore throat* & 14 & $(70.0)$ & 6 & $(27.3)$ & 7 & $(41,2)$ & 0.71 \\
\hline Headache & 18 & $(90.0)$ & 6 & (27.3) & 10 & $(58,8)$ & 0.08 \\
\hline Nasal discharge & 14 & $(70.0)$ & 2 & $(9.1)$ & 5 & $(29,4)$ & 0.15 \\
\hline Diarrhea & 1 & $(5.0)$ & 1 & $(4.5)$ & 4 & $(23,5)$ & 0.81 \\
\hline
\end{tabular}

*All patients with sore throat also had cough. 
Figure 2. Duration of oseltamivir treatment among confirmed cases $(n=20)$ of pandemic A (H1N1) 2009 infection among a population of boarding school pupils in Antananarivo, Madagascar.

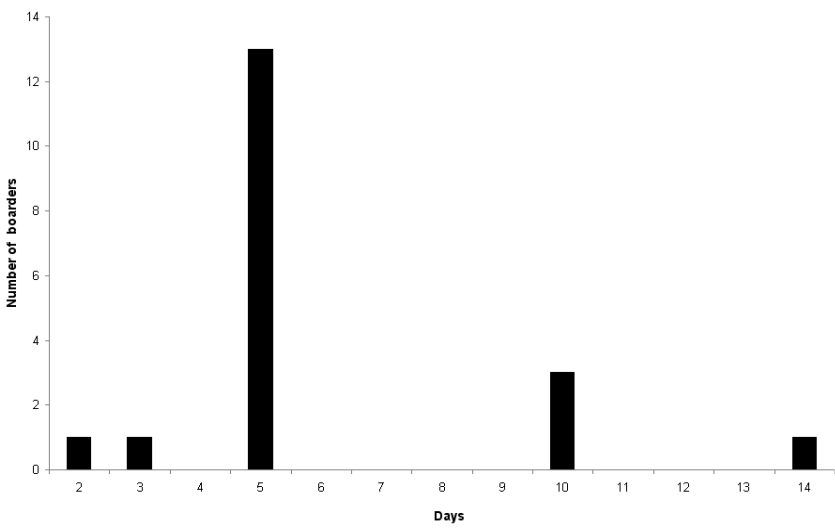

days) according to associated risk factors (e.g. asthma).

Of the 112 contacts or negative cases, eight (7.1\%) did not take oseltamivir prophylaxis, while 75 $(66.9 \%)$ took the medication for at least one week and of them, $63(56.2 \%)$ reported that they had taken the full ten-day course (Figure 3 ).

Among contacts, 38 out of 59 girls (64.4\%) completed the full course compared with 23 of the 43 boys (53.4\%). These differences in compliance according to sex were not significantly different $(\mathrm{n}=$ $102 ; \mathrm{P}=0.2$ ). The course of treatment was completed by $45(61.6 \%)$ of the 73 boarders who did not report any illness in the weeks before or during school closure, and $18(46.1 \%)$ of the 39 boarders who reported any influenza-like symptom (between group $\mathrm{P}=0.11)$.

As shown in Figure 4, the most commonly reported reasons for non-compliance with oseltamivir treatment were as follows: forgetting to take medication $(\mathrm{n}=22)$ and not feeling sick $(\mathrm{n}=15)$. Fifteen boarders reported more than one reason for not taking the tablets. Of the eight children who did not take any doses, three did not feel sick, two were worried about taking it, one did not like taking the medicine, and two did not specify any reason for non-compliance.

\section{Adverse effects of treatment}

Of the 124 children who took at least one oseltamivir tablet, $63(50.8 \%)$ reported at least one symptom associated with adverse effects of oseltamivir therapy, and $46(37.1 \%)$ reported more than one symptom. The frequency of reported symptoms is given in Table 2.

Of the 63 children reporting possible adverse effects, $41(65.1 \%)$ completed the course, compared
Figure 3. Duration of oseltamivir prophylaxis among boarding school pupils in Antananarivo, Madagascar

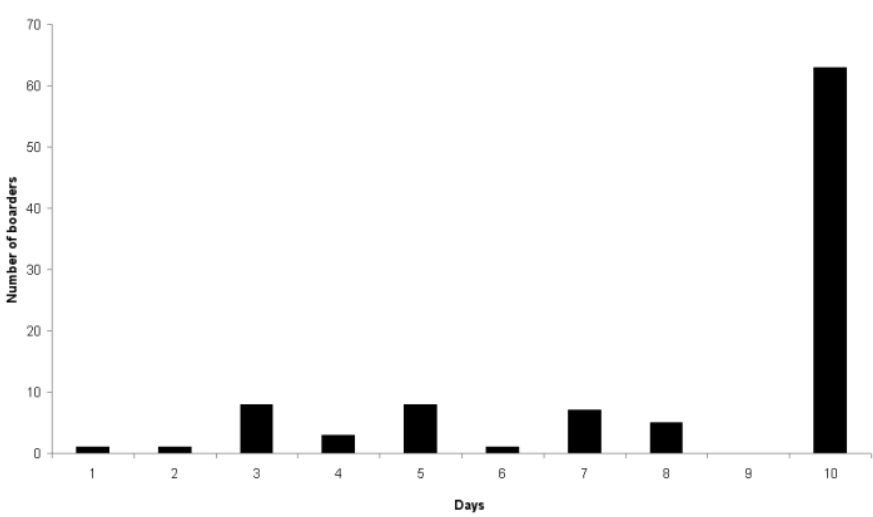

with 35 of the 50 children (70\%) who did not report symptoms (between group $\mathrm{P}=0.6$ ). Nineteen children did not answer this question.

Having at least one adverse effect was not associated with bad compliance $(\mathrm{OR}=1.03 ; 95 \% \mathrm{CI}$ : [0.4-2.5]). No symptom described as an adverse effect was associated with compliance: headache (OR $=1.4 ; 95 \% \mathrm{CI}:[0.5-4.2])$, fatigue $(\mathrm{OR}=1.2 ; 95 \% \mathrm{CI}$ : [0.5-2.9]), difficulty concentrating (OR $1.7 ; 95 \% \mathrm{CI}$ : [0.6-4.9]), sleeping sickness $(\mathrm{OR}=0.7$; $95 \% \mathrm{CI}$ : [0.22.5]).

\section{Discussion}

This study concerns the first reported outbreak of pandemic influenza A(H1N1)2009 virus in Madagascar. We investigated a particular strategy for the 143 boarders in the school that was implemented by medical staff because officials chose not to close the school. All the boarders who were present at school on the day the survey was administered completed the study. This high response rate was largely due to the survey being conducted in the

Table 2. Frequency of different adverse effects among boarding school pupils in Antananarivo, Madagascar who took at least one oseltamivir tablet

\begin{tabular}{lcc}
\hline Symptoms & Number of pupils & Percentage $\mathbf{( \% )}$ \\
\hline Nausea & 10 & $8,1 \%$ \\
Headache & 24 & $19,4 \%$ \\
Tummy ache & 4 & $3,2 \%$ \\
Feeling tired & 48 & $38,7 \%$ \\
Vomiting & 4 & $3,2 \%$ \\
Hard to concentrate & 28 & $22,6 \%$ \\
Sleeping sickness & 15 & $12,1 \%$ \\
Diarrhoea & 2 & $1,6 \%$ \\
Skin rash & 4 & $3,2 \%$ \\
\hline$(\mathrm{n}=124)$ & &
\end{tabular}


Figure 4. Reported reasons among boarding school pupils in Antananarivo Madagascar $(n=57)$ for non-compliance with oseltamivir prophylaxis during October 2009

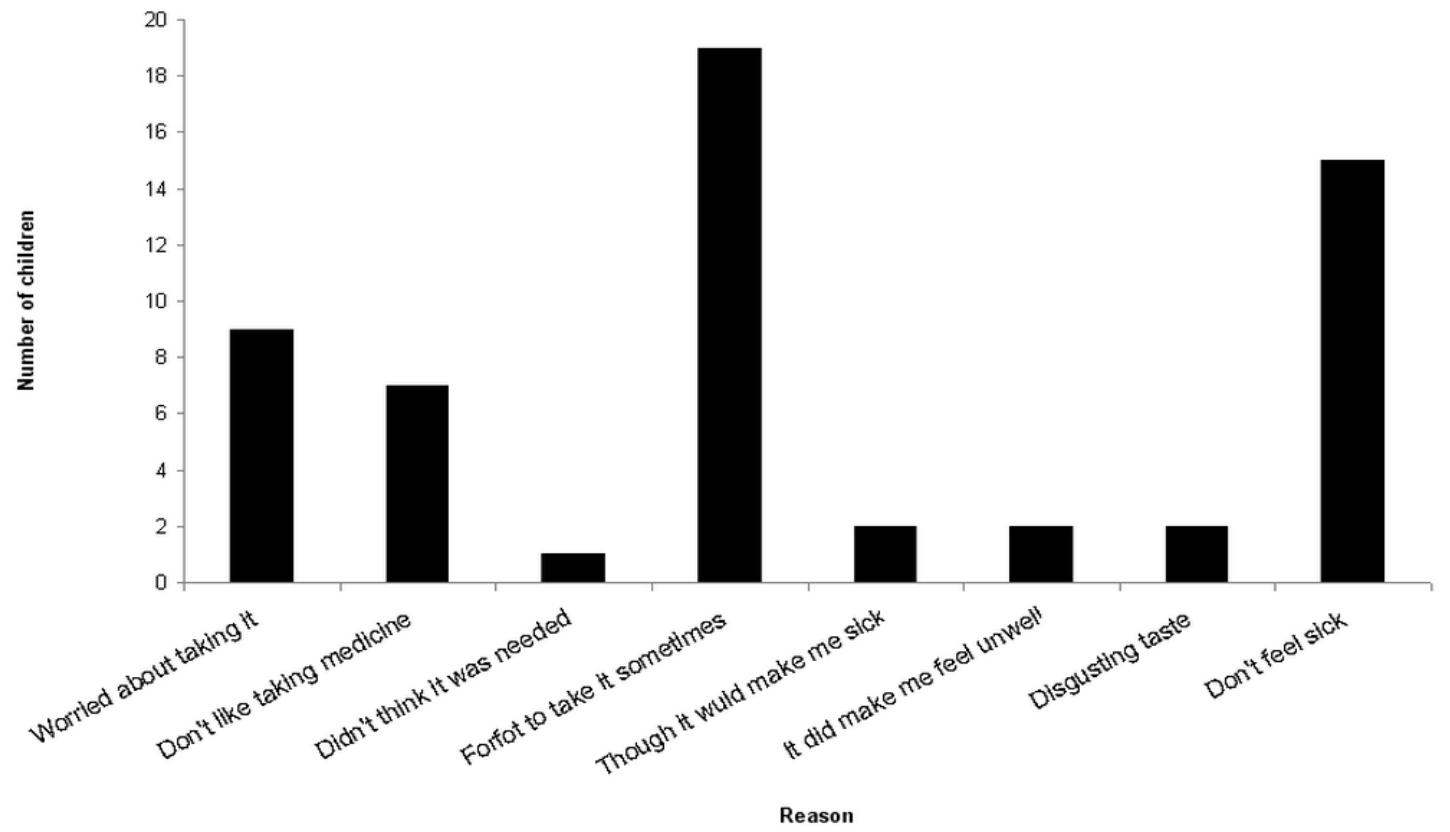

school under supervision. However, completion of the survey in the school may have introduced bias and influenced pupils' answers to the anonymous questionnaire.

All the pupils who reported symptoms associated with pandemic influenza A(H1N1) 2009 infection during the active surveillance period (within seven days of exposure to the last reported case) were tested. After this period, boarders were advised to contact their own general practitioner (GP) if they developed any symptoms. If they presented, it is unlikely that they were tested for pandemic influenza A(H1N1) 2009 infection for the following reasons: all were already aware of the outbreak, their symptoms were relatively mild, and boarders were from areas of Madagascar where relevant laboratory tests were unavailable.

This study provides the first clear evidence of community transmission of the pandemic influenza H1N1 virus within Madagascar. School students are a well-documented source of community influenza transmission and represent markers for more widespread community transmission [13]. In the early stages of a pandemic, schools with a wide geographic catchment area may therefore accelerate the spread of infection. Understanding the epidemiology of pandemic influenza A(H1N1) 2009 virus within schools is crucial to prevent such outbreaks in future. In the present study, the transmission and the severity of illness was consistent with influenza outbreaks in previous seasons [14]. The reported symptoms were also consistent with seasonal influenza although the population of boarders studied was a relatively small sample.

The survey shows that after the first three days of the outbreak and after antiviral prophylaxis for all the boarders, no more boarders were reported ill during the following week. At the same time, attendance rates for the school showed an increase in absenteeism each day. Thus the outbreak was spreading in the overall school population while we observed a decrease within the sub-population of boarders. This also indicates that if transmission occurred first within the school, it subsequently occurred outside over the following weeks.

Our study also suggests that oseltamivir prophylaxis may help to contain outbreaks of influenza. However, a high proportion of schoolchildren might have experienced adverse effects from oseltamivir medication. Over half of those who took the medication reported at least one possible adverse effect including headaches, difficulties concentrating and tiredness. If these symptoms reflect the recognized adverse side effects of oseltamivir prophylaxis, they are higher in frequency compared with the manufacturer's information. When used for prevention purposes, the manufacturer has reported that $18 \%$ of people 
receiving oseltamivir may experience headaches and $8 \%$ may experience asthenia [15]. In some cases who received oseltamivir for prophylaxis, it is possible that symptoms were due to other illnesses and mistakenly attributed to the use of oseltamivir. This explanation is nevertheless unlikely to account for all the symptoms that were experienced during prophylaxis.

Relatively high frequencies of adverse effects of oseltamivir treatment have been reported in a recent study conducted in England [16]. The authors of the study pointed out the young age (11-12 years) of their school population compared with the populations in the original studies that investigated adverse drug effects. However, in our study, the boarders were teenagers (mean age 16.9 yrs), so the age of the study population does not explain these differences. Although the severity of the perceived adverse effects was not assessed, it is likely that most of the symptoms were relatively mild as boarders continued to take the medication.

The findings of our study and those of Wallensten et al. contrast with a Cochrane review on the use of neuraminidase inhibitors to prevent and treat influenza in children. The only adverse effect that was more common than with treatment with placebo was vomiting [17].

Although adverse effects of oseltamivir were common, other concerns, such as the risk of resistance development, should be considered when evaluating the policy of mass prophylactic therapy for novel strains of influenza. This is especially so when the adverse effects are generally mild.

The effectiveness of antiviral prophylaxis is dependent on compliance with the medication. In this study, compliance with oseltamivir prophylaxis was moderate. Compliance was poorer among those who reported symptoms of influenza-like illness, as well as among those who reported symptoms that were likely to have been adverse effects of treatment. This evaluation of oseltamivir chemoprophylaxis among schoolchildren during an outbreak of pandemic influenza $\mathrm{A}(\mathrm{H} 1 \mathrm{~N} 1) 2009$ virus was similar to the evaluation performed by Wallensten et al. [16].The reason compliance was moderate, despite the fact that this was the first school in Madagascar to be affected by pandemic influenza A(H1N1) 2009 virus, may be due to the illness being considered as mild in severity. In turn, this may be affected by many factors such as the severity of the perceived threat of disease, the way in which treatment was offered, and the anticipated and real adverse effects of the medication.

The apparent success in containing the outbreak among the boarding pupils may be linked to the absence of community transmission of the virus, and to the isolation measures that were taken. The success of all intervention also depends on its timing and on the transmission properties of the specific virus strain.

To our knowledge in Madagascar, most illnesses due to pandemic influenza $\mathrm{A}(\mathrm{H} 1 \mathrm{~N} 1) 2009$ infection were generally mild. However, understanding the spread of pandemic influenza A(H1N1) 2009 virus within a school setting and the impact of measures to interrupt transmission is important in helping to prepare for future influenza virus pandemics.

\section{Acknowledgements}

We would like to thank the staff and pupils at the affected school and particularly the staff of the school's health service, Isabelle Rasoarahon and Lydia Veidig.

\section{References}

1. Pan American Health Organization [PAHO] (2004) Final report of the XVI Meeting on Vaccine Preventable-Diseases of the Pan American Health Organization. Washington (District of Columbia): PAHO. Available: http://www.paho.org/English/AD/FCH/IM/TAG16_FinalRe port_2004.pdf. Accessed 19 January 2006.

2. Simonsen L (1999) The global impact of influenza on morbidity and mortality. Vaccine 17: S3-S10.

3. Lessler J, Reich NG, Cummings DAT (2009) Outbreak of 2009 Pandemic Influenza A(H1N1) at a New York City school. N Engl J Med 361: 2628-2636.

4. Calatayud L, Kurkela S, Neave PE, Brock A, Perkins S, Zuckerman, Sudhanva M, Bermingham A, Ellis J, Pebody R, Catchpole M, Heathcock R, Maguire H (2010) Pandemic(H1N1 ${ }^{\circ} 2009$ virus outbreak in a school in London, April-May 2009: an observational study. Epidemiol Infect 138: 183-191.

5. Park SY, Nakata MN, Elm JL, Ching-Lee MR, Rajan R, Giles CA, Hua H, Kanenaka MS, Ando MA, Sasaki JE, Le C, Manuzak A, Wong M, Whelan CA, Ueki R, Kunimoto G, Lee R, Gose R, Chen T, Sreenivasan MV (2010) Outbreak of 2009 Pandemic Influenza A(H1N1) at a school - Hawaii, May 2009. MMWR 58: 1440-1444.

6. Guinard A, Grout L, Durand C, Schwoebel V (2009) Outbreak of influenza $\mathrm{A}(\mathrm{H} 1 \mathrm{~N} 1) \mathrm{v}$ without travel history in a school in the Toulouse district, France, June 2009. Euro Surveill 14: 85-87

7. Kelso JK, Milne GJ, Kelly H (2009) Simulation suggests that rapid activation of social distancing can arrest epidemic development due to a novel strain of influenza. BMC Public Health 9: 117.

8. Heymann AD, Hoch I, Valinsky L, Kokia E, Steinberg DM (2009) School closure may be effective in reducing transmission of respiratory viruses in the community. Epidemiol Infect 137: 1369-1376. 
9. Heymann A, Chodick G, Reichman B, Kokia E, Laufer J (2004) Influence of school closure on the incidence of viral respiratory diseases among children and on health care utilization. Pediatr Infect Dis 23: 675-677.

10. Cowling BJ, Lau EH, Lam CL, Cheng CK, Kovar J, Chan KH, Peiris JS, Leung GM (2008) Effects of school closures, 2008 winter influenza season, Hong Kong. Emerg Infect Dis 14: $1660-1662$.

11. Centers for Disease Control and Prevention protocol of realtime RTPCR for influenza A (H1N1). Available at: http://www.euro.who.int/Document/INF/CDC_realtime_RT PCR_H1N1.pdf. Accessed 11 June 2009.

12. R Development Core Team (2010) R: A language and environment for statistical computing. R Foundation for Statistical Computing, Vienna, Austria. ISBN 3-900051-070, URL http://www.R-project.org. Accessed 11 March 2011.

13. Neuzil KM, Hohlbeinn C, Zhu Y (2002) Illness among schoolchildren during influenza season: effect on school absenteeism, parental absenteeism from work and secondary illness in families. Arch Pediatr Adolesc Med 156: 986-991.

14. Thompson WW, Comanor I, Shay DK (2006) Epidemiology of seasonal influenza: use of surveillance data and statistical modls to estimate the burden of disease. J Inf Dis 1994: S8289.

15. Roche Products Limited (2009) Summary of product characteristics for Tamiflu. The electronic medicines compendium (eMC) website 2009. Available from: http://emc.medicines.org.uk/document.aspx?documentId=10 446. Accessed 11 March 2011.

16. Wallensten A, Oliver I, Lewis D, Harrison S (2009) Compliance and side effects of prophylactic oseltamivir treatment in a school in southwest England. Eurosurveillance 14: 1-4.

17. Matheson NJ, Harnden AR, Perera R, Sheikh A, Symmonds-Abrahams M (2007) Neuraminidase inhibitors for preventing and treating influenza in children. Cochrane Database Syst Rev 2007: CD002744.

\section{Corresponding author}

Vincent Richard

Epidemiologic Unit

Institut Pasteur from Madagascar

B.P. 1274

Ambatofotsikely

101 Antananarivo, Madagascar

Email: vrichard@ pasteur.mg

Conflict of interests: No conflict of interests is declared. 